\title{
Research on Engineering Construction Safety Integration based on BIM and RFID
}

\author{
Li Binyong ${ }^{1}$, Du Zeyan ${ }^{2,3, a}$, Xie Dayong and Huang Lang ${ }^{2}$ \\ ${ }^{1}$ College of Cybersecurity, Chengdu University of Information Technology, 610225 Chengdu, China \\ ${ }^{2}$ College of Yinxing Hospitality Management, Chengdu University of Information Technology, 611743 Chengdu, China \\ ${ }^{3}$ College of Management and Economics, Kunming University of Science and Technology, 650093 Kunming, China
}

\begin{abstract}
According to the fact that the traditional manual management method can not solve the problem of frequent accidents in the engineering construction process, the integrated scheme of safety prevention based on BIM and RFID is put forward, and the principle and technical feasibility of the integrated scheme are analyzed. Based on the integration technology scheme, the multi-layer architecture design pattern is adopted, and the software hierarchy of the integration system is designed in detail. On this basis, the integrated application network for engineering construction safety control is analyzed and designed by using the technology based on BIM and RFID. The research results of this paper provide a feasible technical reference scheme for the automatic identification and dynamic monitoring and early warning of the hidden dangers in the construction site.
\end{abstract}

\section{Introduction}

Engineering safety has always been the top priority of the Chinese government in the supervision of production safety. In recent years, with the rapid development of engineering construction in China, it also leads to frequent accidents in construction engineering. The traditional safety management mode not only consumes a lot of manpower, material resources and financial resources, but also is difficult to effectively prevent all kinds of potential safety hidden dangers in the construction process. The construction of engineering safety in China has always been faced with severe challenges of safety supervision, and the construction of engineering has been in the situation of not optimistic for a long time. Especially in recent years, with the implementation of Belt and Road strategy, the construction safety of overseas cooperative investment has become the focus of concern and concern in the country along the road of Belt and Road.

While it is more and more difficult to satisfy the safety management of engineering construction by traditional means, it is urgent to combine modern information technology to ensure the safety of engineering construction. With the rise of BIM(Building Information Modeling), it not only completely changes the management mode of construction engineering, but also promotes the technological innovation in the field of engineering construction. The virtualization of construction site and the dynamic programming of all kinds of safety elements can be realized effectively by BIM technology. At the same time, the use of RFID(Radio Frequency Identification) technology can effectively support the real-time collection and processing of all kinds of information of safety elements in construction site, in order to achieve the goal of realtime monitoring of construction site.

It is not difficult to predict that the combination of two kinds of new information technology means BIM and RFID will effectively avoid and reduce the occurrence of various kinds of safety accidents in the process of engineering construction.

\section{Integration scheme based on BIM and RFID}

In order to meet the requirements of automatic identification and dynamic monitoring of hidden safety problems in engineering construction site, this paper presents an integrated scheme as shown in figure 1. This scheme combines the modern Internet of things technology with the building information model, and integrates the technology scheme based on RFID and BIM.

The application principle of this technical scheme can be described as follows: by deploying RFID electronic tag, it can form an Internet of things with the people, materials and objects in the construction site of the project, and use RFID radio frequency identification technology. The system can collect all kinds of safety hidden trouble information in the construction site dynamically and in real time. At the same time, through the systematic modeling of structure information, entity attribute information, building information and early warning parameters, the BIM model base covering all kinds of resource elements in engineering construction

\footnotetext{
a Corresponding author: lby@cuit.edu.cn
} 
site is formed. On this basis, the software program is developed to support the dynamic identification and early warning of the potential safety hazard in the construction site.

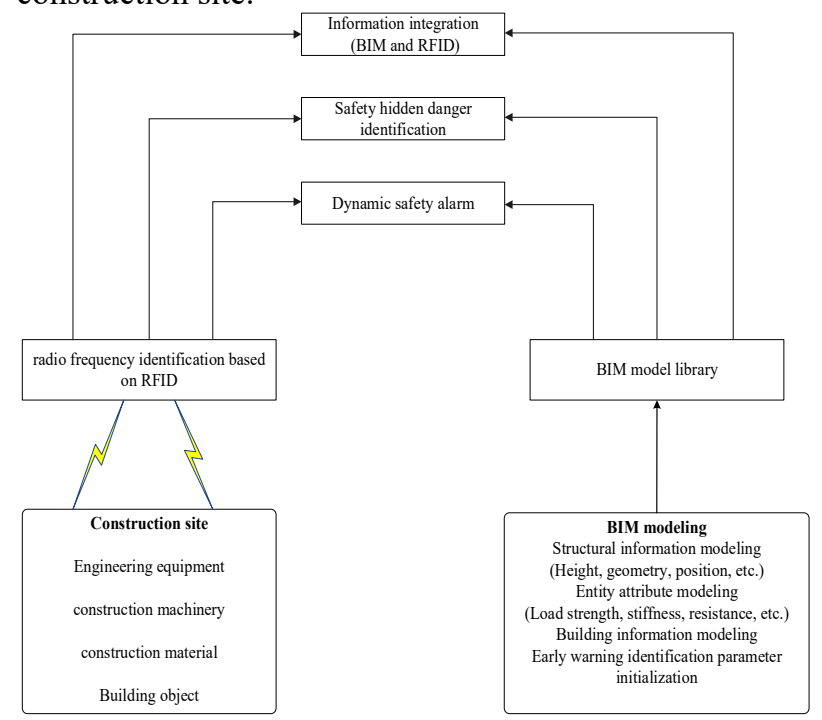

Figure 1. Integration scheme based on BIM and FRID

\section{Software hierarchical architecture integration design}

Whether the software system design is reasonable or not is directly related to the smooth interaction of the network information flow of the engineering construction integration, so the software system is the core of the information processing system to ensure the engineering construction safety.

At the same time, the software architecture is designed from the perspective of hierarchy, and the low coupling, extensibility and reusability of the software are taken into account. In this paper, the multi-tier architecture design pattern is adopted, and the whole system is designed as the software hierarchical integration system as shown in figure 2 .

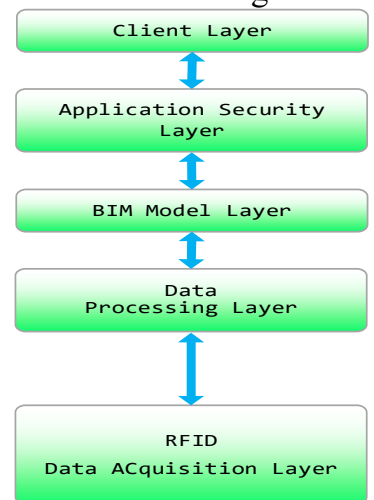

Figure 2. Software hierarchy design

In the above design ideas, the software system, which is called from layer to layer and connected in turn, not only clarifies the functions of each layer of software, but also ensures the independence and loose coupling of each layer of software.
This layer is the top layer of software, which is responsible for interacting with users, facing project manager, constructor, equipment manager, chief engineer, supervisor, tower crane staff, etc. The current mainstream .NET Framework or J2EE Framework can be used to support the temporary of Web visual application interface, and DIV CSS technology can be used to control the display style of the page, so as to provide a friendly human-computer interface for users. Through this layer, users can obtain or identify all kinds of hazard information in the engineering site in real time, so as to prevent and control the potential hidden danger in time.

\subsection{Application security layer}

The application of the safety layer is mainly responsible for automatic identification and early warning of the hidden dangers in the construction site. It needs data processing layer BIM model layer and RFID data acquisition layer to support its application security identification and processing and provide dynamic realtime early warning support for the user layer.

In particular, it relies on RFID technology, through the static layout analysis and dynamic movement detection of the personnel, materials, machinery and other related entities in the construction site, and makes use of the prediction simulation and collision detection algorithms of the construction scene, etc. To realize the dynamic identification of construction site, construction layout, construction hazard source and construction hidden trouble based on BIM, and to provide early warning or alarm service for user front end in time, in order to ensure the safety of the whole construction process.

\subsection{BIM model layer}

This layer can be regarded as a resource integration layer, which mainly integrates all kinds of construction information resources, structural information resources, dangerous construction areas and so on, and is stored in the BIM database through the form of BIM building information model.

Moreover, the layer should build BIM building information model from the view of safety. It needs to focus on the safety attributes such as load strength, stiffness and resistance of integrated BIM resource model, and combine its spatial location, height, geometric size and other resource attributes. Through 3D dynamic simulation, the dynamic reorganization and organization of BIM resource elements are realized, in order to provide directly related data source support for construction safety data analysis of data processing layer. The security hidden trouble identification of the security layer has not been applied to provide reliable decision support.

\subsection{Client layer}




\subsection{Data processing layer}

The data processing layer is the bridge between the model layer and the data acquisition layer. The main function of the data processing layer is to integrate and integrate the data collected by the BIM model base and the data collected by RFID, and on this basis, In view of the related hidden safety problems that may exist or appear in the process of engineering construction, combined with the preset parameters, the comprehensive analysis and processing of the data are carried out.

Among them, it mainly involves the cooperative association of BIM model data and the data collected by RFID, cooperative grouping, cooperative checking, collaborative filtering, collaborative fusion, collaborative contrast, etc., in order to identify and cross-match the data sources that accord with the characteristics of hidden security problems. The system can generate the pre-data source to meet the engineering construction safety warning.

\subsection{Data acquisition layer}

This layer mainly carries on the data collection through the RFID electron to the engineering construction site, including the static data acquisition and the dynamic data acquisition. The static data acquisition mainly refers to the layout and coordinates of the static objects in the construction site, while the dynamic data acquisition mainly refers to the dynamic moving data collection of the people, materials and objects on the construction site in order to locate the moving entities in real time. Provides data source support for potential collision risks.

The engineering field data acquisition based on RFID needs to rely on RFID reader to recognize RFID tag information in real time. Before data acquisition, the RFID tag should be laid out first, usually the RFID tag can be attached to the target object, at the same time, the RFID tag should be initialized and defined. It includes the ID, of RFID tag and the basic attribute of field component corresponding to ID tag.

\section{Integrated application based on BIM and RFID}

The purpose of integrating two kinds of modern information technology, BIM and FRID, is to identify and warn the safety of engineering construction site more effectively and comprehensively.

As shown in figure 3, by posting or embedding RFID tags on the surface or inside of construction machinery, buildings, building materials, etc., at the construction site, the data of RFID tags are read through RFID readers, Using RFID wireless radio frequency transmission technology and API interface, real-time transmission of all kinds of information from the engineering site to the integrated monitoring and processing center is carried out in order to realize the dynamic collection of the environmental data of the project site by the integrated monitoring center system.

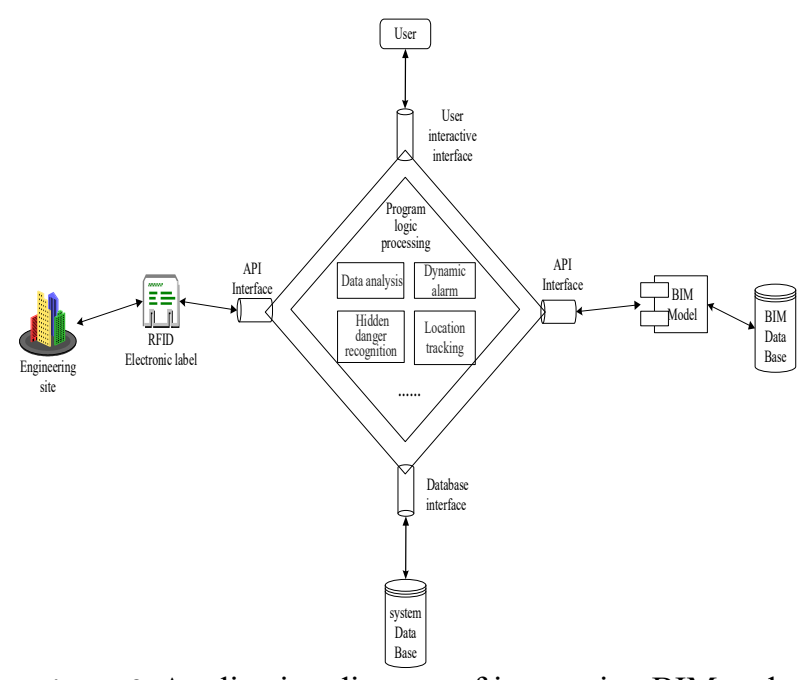

Figure 3. Application diagram of integrating BIM and RFID technology

At the same time, in order to realize the unified coordination and management of the whole life cycle stages, such as engineering design, construction, operation management and so on, it is necessary to simulate all kinds of physical resource elements involved in building engineering through digital 3D technology. The BIM information model is constructed and stored in the BIM database. On this basis, the API interface is used to realize and integrate the information model call and interaction of the monitoring center.

Finally, the integrated monitoring center uses the information network to analyze and process the information collected by the RFID tag and the data of the BIM model, and stores the processing results to the system database. In order to provide data source support for the hidden trouble identification, dynamic early warning and location tracking in the following construction process.

\section{Conclusions}

Engineering construction is a high-risk area with frequent safety accidents. In the traditional construction safety control and management means more and more overstretched, the use of modern information technology to support the construction site security management, there is no doubt efficient and reliable advantages and significance. Based on the combination of RFID and $\mathrm{BIM}$, this paper constructs the technology scheme of integration of the Internet of things based on RFID and the building model base based on BIM, so as to locate and identify the source of the potential safety hazard in the construction site. And intelligent early warning provides feasible technical support, at the same time to improve the quality and efficiency of engineering safety management, also has certain guiding significance.

\section{Acknowledgements}

This work was financially supported by the National Natural Science Foundation of China(Grant No. 71701026) and Sichuan Science and Technology 
Program (Grant No. 2018GZ0307) and the Key projects of Sichuan Provincial Education Department(Grant No. 17ZA0069) and the soft science project of Chengdu in China(Grant No. 2016-RK00-00089-ZF).

\section{References}

1. Q Liu, Z.M Su, S.Y Zu. Journal of civil engineering and management. Chn. J. 33, 5(2016)

2. Y.G Chen, H.L Shan. Scientific and technological bulletin. Chn. J. 32, 7(2016)

3. Q.W Shi, Y.S Pang, H.Y Jiang. Journal of civil engineering and management. Chn. J. 33, 2(2016)

4. J.J Zhang, Y Han, G.X Ma, S.C Han. Journal of engineering management. Chn. J. 29, 6(2015)

5. F Li, W Li, Z Liu, Z Li. Information technology of civil construction engineering . Chn. J. 7, 5(2015)

6. Y Zai, N Li, X.Q Ai, W He. Construction technique . Chn. J. 44, 12(2015)

7. H.L Guo, Y.T Yu, W.P Liu, W.S Zhang. Journal of engineering management Chn. J. 28, 4(2014) 